Article

\title{
Towards New Constraints in Extended Theories of Gravity: Cosmography and Gravitational-Wave Signals from Neutron Stars
}

\author{
Álvaro de la Cruz Dombriz \\ Cosmology and Gravity Group, Mathematics and Applied Mathematics Department, University of Cape Town, \\ Cape Town 7700, South Africa \\ * Correspondence: alvaro.delacruzdombriz@uct.ac.za
}

Received: 29 January 2018; Accepted: 24 February 2018; Published: 27 February 2018

\begin{abstract}
Combined cosmological, astrophysical and numerical tests may shed some light on the viability of theories of gravity beyond Einsteinian relativity. In this letter, we present two different techniques providing complementary ways of testing new physics beyond the $\Lambda \mathrm{CDM}$ cosmological paradigm. First, we shall present some of the latest progress and shortcomings in the cosmographic model-independent approach for several modified gravity theories using supernovae catalogues, baryonic acoustic oscillation data and $H(z)$ differential age compilations. Second, we shall show how once the Einsteinian paradigm is abandoned, the phenomenology of neutron stars changes dramatically since neutron-star masses can be much larger than their General Relativity counterparts. Consequently, the total energy available for radiating gravitational waves could be of the order of several solar masses, and thus a merger of these stars constitutes a privileged wave source. Unfortunately at the present time our persisting lack of understanding in the strong interaction sector does not allow to distinguish the alternative theories from the usual General Relativity predictions.
\end{abstract}

Keywords: extended theories of gravity; cosmography; neutron stars; gravitational waves

\section{Introduction}

The discovery of the accelerated expansion of the universe [1-3], the more recent detection of gravitational waves as emitted from black-hole and neutron-star mergers [4-10] and the increased precision in relativistic effects in the growth of large-scale structures [11-14] have propelled the intensive study of extended (also dubbed modified) theories of gravity. Roughly speaking, the whole pleyade of such theories generally aims to overcome the limitations that the cosmological Concordance $\Lambda \mathrm{CDM}$ model suffers when applied to several astrophysical and cosmological tests in a broad range of energies, from the Planck scale - and the lack of a consistent quantum field theory of gravity - to the late-time cosmological scales-and the lack of explanation for observed acceleration. Many of these proposals [15-19] aim to avoid the need of the usually invoked dark components, either through a mere cosmological constant, as in the $\Lambda \mathrm{CDM}$ model, where dark energy is nothing but a cosmological constant, or through artificial scalar fields which are added to the usual General Relativity (GR) term, as in phantom/quintessence-like models.

Since the number of viable modified gravities at hand remain sufficiently large, complementary techniques in both astrophysical and cosmological scales as well as in strong-gravity regimes, where GR has never been directly tested, are the ideal tools to compare theoretical predictions with catalogues of dataset, extracted, for instance, from both the cosmic microwave background, cosmological expansion, large-scale structures, astrophysical configurations and gravitational-wave signals. Usually, such comparisons suffer from the so-called degeneracy problem [20], meaning that several competitive gravitational theories are capable of explaining the same phenomena with roughly speaking the same 
statistical significance. The breaking of such a problem with complementary tests is precisely the leit-motiv of this communication.

Among competitive extended theories of gravity, some of the most successful ones include the so-called scalar-tensor $f(R)$ theories, where the gravitational Lagrangian includes powers of the Ricci scalar $R$ encompassed in an arbitrary function of $R$ (c.f. [21-25] for extensive reviews on the subject); or extended theories of teleparallel gravity $F(\mathcal{T})(c . f$. [26-38] for results in the subject) where the covariant action is written in terms of an arbitrary function of the torsion scalar $\mathcal{T}$, which indeed extends the usual teleparallel gravity approach.

The paper is organised as follows, in Section 2 we present the rudiments and main results when model-independent (cosmographic) techniques are used to reconstruct $f(R)$ and $F(\mathcal{T})$ Lagrangians capable of causing the observed cosmological evolution once the Copernican principle is assumed. Then, in Section 3 we shall present the main features of neutron stars for paradigmatic quadratic $f(R)$ theories with no intermediate approximation in the system of equations. Analysis will show that distant observers would observe a gravitational mass greater than the GR counterpart for the same particle-physics equation of state, the exterior solution asymptotically-although not exactly-being Schwarzschild-like. Finally, we conclude the paper by presenting our main conclusions in Section 4 as well as some prospects to improve the aforementioned analyses.

\section{Cosmography}

A possible way of alleviating the aforementioned degeneracy consists of combining different cosmological measurements in order to reduce the phase space of free parameters. Nevertheless, the caveat of any measurement is the implicit a priori assumption about the statistical validity of a given cosmological model. For that reason, amongst several statistical treatments, model-independent techniques aim to guarantee that the statistical outcomes do not depend upon the choice of the model itself. Cosmography of the Universe indeed belongs to this class of techniques [39]. Cosmographic studies in extended theories have been limited to recent research [40,41] proving how much disfavoured such a technique is in comparison with Gaussian processes of reconstruction [42].

Nonetheless, there were indeed seminal references for cosmographic techniques in the context of $f(R)$ theories included $[40,41,43,44]$, whereas for $F(\mathcal{T})$ they were restricted to [45]. In our recent investigations [46] we expanded each class of extended theory under consideration in powers of redshift $z$ around the present time, making no additional assumptions on the values of the theory's parameters at the present epoch. In [46] we indeed combined data from the Union 2.1 SNIa catalogue, BAO data and $H(z)$ differential age compilations with the support of the most recent Planck data, so the cosmological expansion can be tested on different epochs of the cosmological evolution. There, we performed a Monte Carlo analysis using a Metropolis-Hastings algorithm with a Gelman-Rubin convergence criterion and flat priors ${ }^{1}$, reporting $1-\sigma$ and $2-\sigma$ confidence levels. Our results showed how previous analyses were unable to capture essential trends in the considered theories and led to underestimation of the cosmographic bounds. In order to show the dependence of the results with the considered redshift interval, we first used data in the very small redshift regime $z<1$. We then performed the same analyses but with all data points for every dataset, i.e., without limiting them to $z<1^{2}$. For $f(R)$ theories, we found that all model parameters were compatible with zero at about the 1- $\sigma$ level, albeit the shapes of contour plots were different from pure ellipses, a fact related to the higher-order character of these theories. In addition, such shapes change as one shifts from the $z<1$ analysis to the all- $z$ analysis. Main results are shown in Figure 1. For $F(\mathcal{T})$ theories we again found that all model parameters are compatible with zero at about the $1-\sigma$ level, while the $2-\sigma$ level

with the exception of $r_{\mathrm{s}}\left(z_{\mathrm{drag}}\right)$, in which a Gaussian prior was used and set at Planck's best values [46].

This does not apply to BAO measurements, since all data points are confined inside $z<1$. Hence, the same data were used for both fits [46]. 
enables larger parameter ranges, especially for higher derivatives. In fact, dimensionless parameters proportional to the third and fourth derivatives of $F(\mathcal{T})$ turned out to be only constrained at the level of $\mathcal{O}\left(10^{2}\right)$ and $\mathcal{O}\left(10^{2}\right)$ levels for $z<1$ data, and $\mathcal{O}(1)$ and $\mathcal{O}(10)$ for the full dataset [46]. The best-fit points, still relatively close to model parameters, were compatible with zero, although the posterior probabilities are far from being Gaussian. Main results are shown in Figure 2. In both Figures 1 and 2, $h_{0} \equiv H\left(z_{\text {today }}\right) /\left(100 \mathrm{~km} \mathrm{~s}^{-1} \mathrm{Mpc}^{-1}\right)$ and $\Delta_{M}$ holds for the luminosity distance nuisance parameter defined as $\mu^{\text {fit }}\left(z_{i}\right)=\mu^{\text {Union2.1 }}\left(z_{i}\right)+\Delta_{M}$ which depends on $H_{0}$ and $M$.

Finally, for those two theories, we also compared our statistical results to standard criteria for statistical significance, namely the AIC and BIC criteria. This discussion enabled us to state that when expanding the standard $\Lambda \mathrm{CDM}$ cosmological model to the third order, the corresponding third orders for both $f(R)$ and $F(\mathcal{T})$ theories turn out to be statistically favoured by using the AIC criteria, however disfavoured in the case of BIC. For further details refer to Section 5.6 in [46] in Table 8 therein.

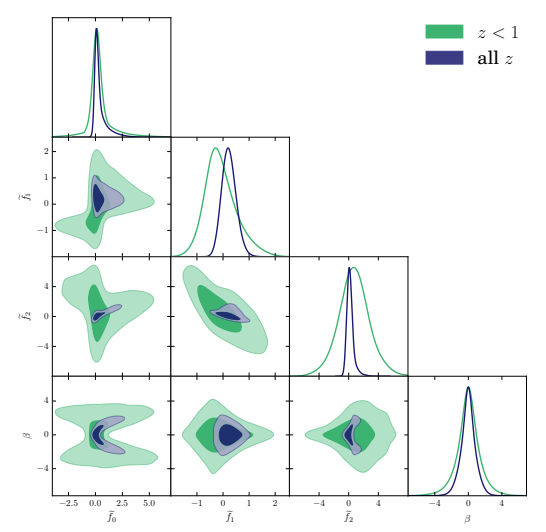

\begin{tabular}{ccc} 
Parameter & $\mathbf{z}<\mathbf{1}$ & All-z \\
\hline$h_{0}$ & $69.9_{-1.9}^{+2.0}(69.6)$ & $69.6_{-1.8}^{+1.8}(69.3)$ \\
$\Delta_{M}$ & $-0.006_{-0.048}^{+0.048}(-0.017)$ & $-0.007_{-0.045}^{+0.045}(-0.018)$ \\
$\widetilde{\Omega}_{m}$ & $0.292_{-0.060}^{+0.063}(0.284)$ & $0.293_{-0.049}^{+0.053}(0.292)$ \\
$\widetilde{f}_{0}$ & $0.4_{-2.3}^{+3.5}(4.63)$ & $0.40_{-0.70}^{+1.5}(0.348)$ \\
$\widetilde{f}_{1}$ & $-0.1_{-1.1}^{+1.3}(-0.017)$ & $0.23_{-0.53}^{+0.55}(0.300)$ \\
$\widetilde{f}_{2}$ & $0.7_{-4.3}^{+4.5}(1.95)$ & $0.20_{-0.86}^{+1.0}(0.074)$ \\
$\beta$ & $0.0_{-3.0}^{+3.0}(-3.951)$ & $0.0_{-1.8}^{+1.8}(0.711)$ \\
$\chi_{\min }^{2}$ & $552.1 /(581$ d.o.f. $)$ & $585.6 /(616$ d.o.f. $)$ \\
\hline
\end{tabular}

Figure 1. Results for $f(R)$ theories for $z<1$ and all-z as depicted in [46]. Errors on this table and on Figure 2 correspond to $95 \%$ confidence levels and best fit values are indicated in brackets. The model parameters lie centered around zero, although order one departures are spotted. $f(R)$ parameters contours shapes show how the likelihood surface differs from a multi-variate Gaussian even in the vicinity of the maximum likelihood point. The following variables have been used: $\alpha=\left.f_{R}\right|_{z=0}$, $\tilde{\Omega}_{m}=\frac{\Omega_{m}}{\alpha}, \tilde{f}_{0}=\left.\frac{1}{6 H_{0}^{2} \alpha} f\right|_{z=0}-1+\frac{\Omega_{m}}{2}, \tilde{f}_{1}=\left.\frac{1}{6 H_{0}^{2} \alpha} \frac{\partial f}{\partial z}\right|_{z=0}-\frac{3 \Omega_{m}}{2}, \tilde{f}_{2}=\left.\frac{1}{6 H_{0}^{2} \alpha} \frac{\partial^{2} f}{\partial z^{2}}\right|_{z=0}-3 \Omega_{m}$ and $\beta^{2}=$ $\left.\frac{6 H_{0}^{2}}{\alpha} f_{R R}\right|_{z=0}$.

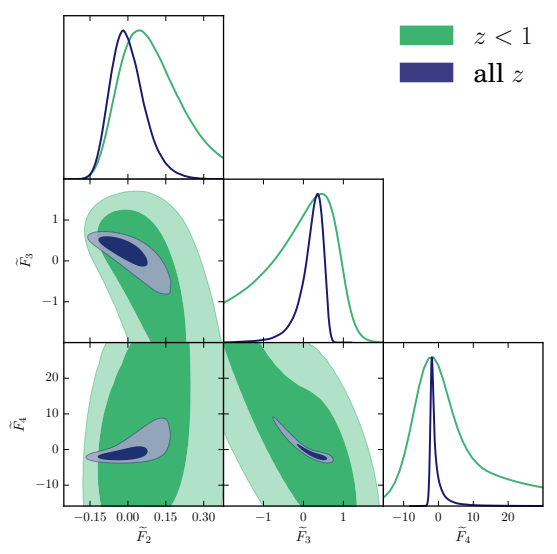

\begin{tabular}{ccc} 
Parameter & $\mathbf{z}<\mathbf{1}$ & All-z \\
\hline$h_{0}$ & $70.1_{-1.9}^{+1.9}(69.2)$ & $69.8_{-1.8}^{+1.8}(69.2)$ \\
$\Delta_{M}$ & $-0.002_{-0.047}^{+0.047}(-0.023)$ & $-0.004_{-0.044}^{+0.044}(-0.020)$ \\
$\widetilde{\Omega}_{m}$ & $0.294_{-0.058}^{+0.062}(0.294)$ & $0.294_{-0.047}^{+0.052}(0.289)$ \\
$\widetilde{F}_{2}$ & $0.11_{-0.23}^{+0.28}(0.029)$ & $0.00_{-0.13}^{+0.14}(-0.024)$ \\
$\widetilde{F}_{3}$ & $-1.3_{-5.4}^{+2.7}(0.028)$ & $0.18_{-0.68}^{+0.49}(0.293)$ \\
$\widetilde{F}_{4}$ & $42_{-70}^{+200}(-0.66)$ & $-0.5_{-2.7}^{+5.6}(-1.636)$ \\
$\chi_{\min }^{2}$ & $552.2 /(581$ d.o.f. $)$ & $585.6 /(616$ d.o.f. $)$ \\
\hline
\end{tabular}

Figure 2. Results for $F(\mathcal{T})$ theories for $z<1$ and all-z as depicted in [46]. As can be seen, all model parameters are highly compatible with zero (i.e., with the $\Lambda$ CDM limit), but $\widetilde{F}_{3}$ and especially $\widetilde{F}_{4}$ can deviate rather substantially from zero, particularly for the $z<1$ analysis. Note that $\widetilde{F}_{j}=F_{j} / F_{1} \quad(j=2,3,4)$, with $F_{i}=\left.\mathcal{T}^{i-1} \frac{\partial^{i} F}{\partial \mathcal{T}^{i}}\right|_{z=0}$. 


\section{Spherical Configurations: Neutron Stars}

As stated in the introduction, a window of particular interest in shedding light on the nature of the gravitational interaction lies in high-energy configurations such as black holes ${ }^{3}$ as well as relativistic (neutron and quark) stars. In such strong gravitational field regimes, predictions of extended theories may complement the gravitational picture as provided by the low-curvature cosmological evolution at late times. Herein, we shall illustrate how for viable classes of $f(R)$ Lagrangians, the relations between masses and radii parameters of relativistic stars differ from their Einsteinian counterparts dramatically.

As a straightforward consequence of the Tolman-Oppenheimer-Volkoff (TOV) [47] analysis, once GR is assumed as the correct theory of gravity, there are certainly upper limits for realistic neutron star masses, being the theoretical mass limit itself increased along the years, from $\mathcal{O}\left(0.6 M_{\odot}\right)$ for a free neutron gas equation of state $(\mathrm{EoS})$, to $\mathcal{O}\left(2.2 \mathrm{M}_{\odot}\right)$ limits imposed by stiffer chiral interactions [48]. Thanks to the discovery of a neutron star with mass $1.974 M_{\odot}[49]$ - confirmed later on by other measurements [50-53], the precise sighting [54] through binary system measurements, double neutron stars features and pulsar systems [55], the validity of GR standard predictions have been indeed put into question. Thus the interest for such configurations in extended theories of gravity, mainly in scalar-tensor theories [56-71], has aimed to reconcile predictions with astrophysical results.

The approach we followed in both $[68,72]$ was novel since for static and spherically symmetric configurations (a) instead of assuming any perturbative analysis, the exact higher-order system of differential equations was solved; and $(b)$ the Schwarzschild solution was not assumed to be exactly—but asymptotically—-the exterior solution ${ }^{4}$. Consequently, the star outer spacetime may also contribute to the total gravitational mass. Thus, the emergence of the so-called gravitational sphere around the star provides an extra contribution to the gravitational mass as measured by distant observers. Moreover, the obtained exterior solution crucially depends upon the chosen nuclear matter EoS because the latter determines the value of the Ricci scalar at the star surface [68] and therefore the amplitude of its damped oscillations.

In order to illustrate the power of this analysis, we present herein the main features of neutron stars in the context of the paradigmatic $f(R)=R+\alpha R^{2}$ gravity $^{5}$ and the well-known AP4 equation of state [75] which hosts a three-nucleon potential and Argonne 18 potential with UIX potential. Indeed, for $\alpha>0$, the chosen initial condition for $R(r=0)$ must guarantee that the Schwarzschild solution is asymptotically approached at spatial infinity ${ }^{6}$. The performed analysis proved that for a given density at the centre of the star, the stellar mass is indeed smaller than the GR counterpart. As a result, the gravitational mass overcomes the GR counterpart for central density values higher than a given critical central density. This effect was noticed for several EoS, including the case of quark stars as can be found in [72]. Results are presented in Figures 3 and 4 where both GR and $f(R)$ quadratic predictions are depicted.

3 c.f. [25] for a review about the existence and main features of black holes in theories beyond GR.

4 In the context of $f(R)$ theories, it is a well-known fact that the matching conditions at the edge of the star do not impose the Ricci curvature to vanish [73]. This fact constitutes a straightforward violation of the Jebsen-Birkhoff theorem in $f(R)$ theories $[73,74]$.

5 c.f. [68] for a thorough analysis regarding the so-called $f(R)$ Hu-Sawicki model.

6 For $\alpha<0$, results in [72] concluded that, although outside the star the Ricci scalar shows damped oscillations, the total gravitational mass would increase with the radial distance, showing the lack of realism of such an $\alpha$ parameter space. 

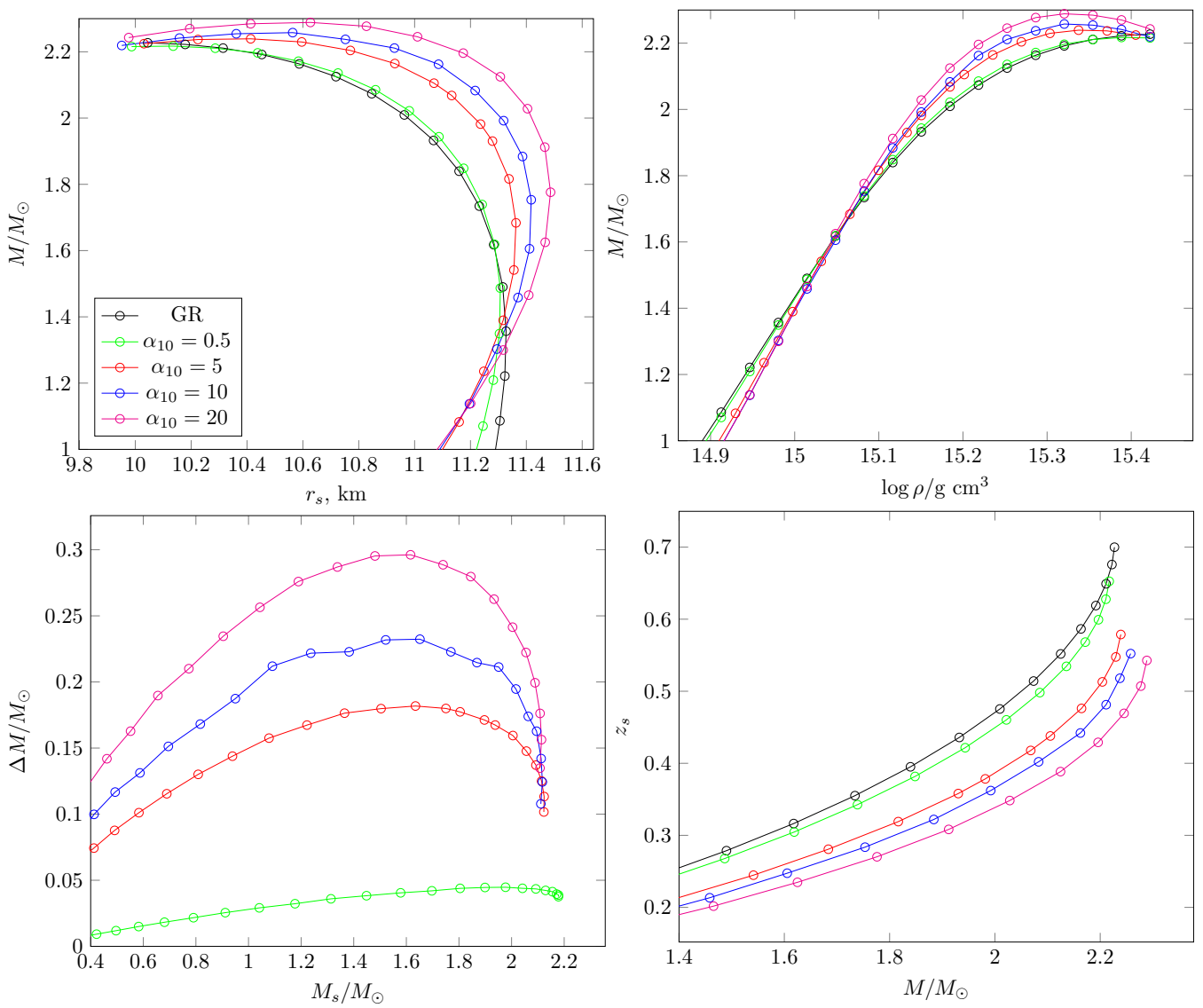

Figure 3. Upper panel: relation between the gravitational mass $(M)$ and radius of the star for AP4 EoS in $R^{2}$-gravity when compared with the General Relativity predictions (left); relation between the gravitational mass and the central density of the star (right). The symbol $\alpha_{10}$ refers to $\alpha \times 10^{10} \mathrm{~cm}^{2}$. Lower panel: the dependence of the contribution of gravitational sphere into gravitational mass $\Delta M=M-M_{s}$ with stellar mass $M_{s} \equiv m\left(r_{\text {surface }}\right)$ (left); surface gravitational redshift $z_{s}$ defined as $z_{S}=\left(1-2 M_{S} / r_{S}\right)^{-1 / 2}-1$, where $M_{S}$ holds for the stellar mass, as a function of gravitational mass (right).
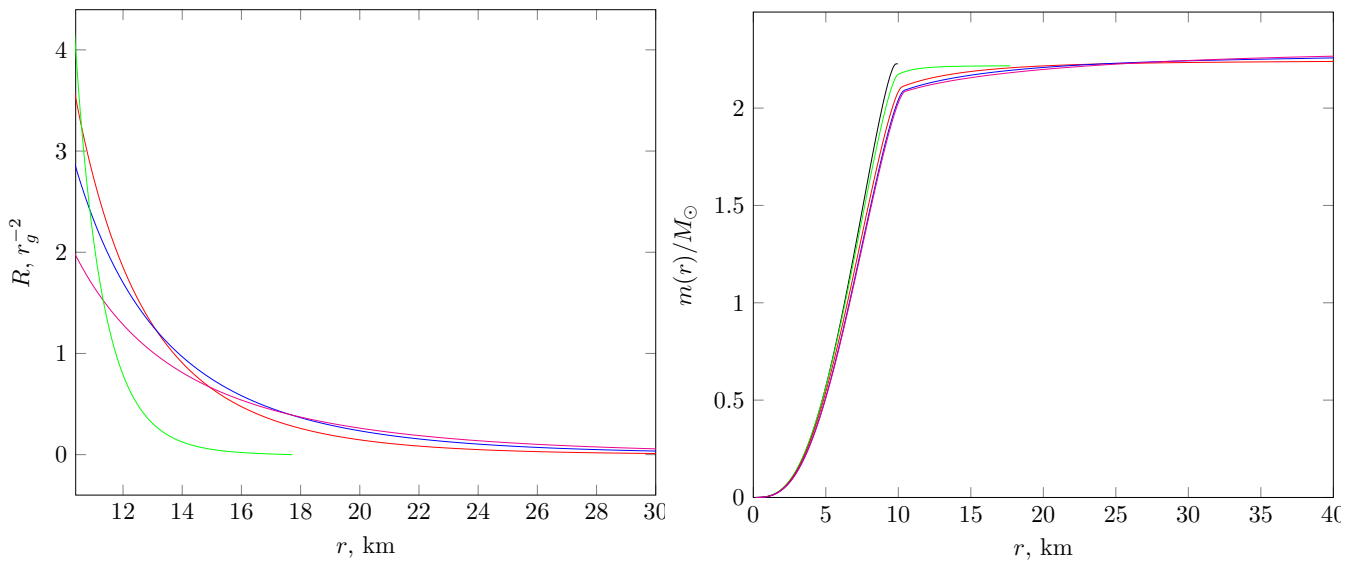

Figure 4. Left panel: Dependence of the scalar curvature outside the star for stellar configurations with maximal mass for AP4 EoS. Scalar curvature is given in units of $\left.r_{g}^{-2} \equiv c^{4} / G^{2} M_{\odot}^{2}\right)$. Right panel: the stellar mass profile $m(r)$ for stellar configurations with maximal gravitational mass. 


\section{Conclusions}

In this communication we have presented a brief overview of two powerful techniques which are called to shed light on the underlying nature of the gravitational interaction, namely model-independent cosmographic methods, targeting the framework of two extensions of General Relativity, and the main features of compact neutron stars with a paradigmatic realistic equation of state in scalar-tensor theories.

First, our cosmographic analysis was only based on the most general assumptions. Our results noticed discrepancies with previous literature for several classes of reconstructed extended dark-energy theories. In particular, according to the statistical results presented above for the cosmographic analysis, the reconstructed gravitational Lagrangians do not need to exactly mimic the standard linear dependence with the Ricci scalar at present times. Obviously, further work is needed so this technique becomes more competitive. For instance, future analyses must take higher-redshift catalogues into account, desirably with different redshift windows of data points. Moreover, there are still several challenges faced by this method to be addressed. In particular: (i) the need for a clear definition of the ideal auxiliary variable, their range of applicability and a thorough testing against mock data; (ii) the definition of a robust statistical method-if any-in order to establish a trade-off between number of data points, number of cosmographic parameters and the required statistical evidence to safely rule out models; and (iii) motivated statistical priors over the extra parameters in higher-order theories in order to get competitive constraints for these theories.

Complementary, once theory-dependent extensions of General Relativity are considered, the study of compact stars remains as a mandatory task in order to extract novel theoretical and observational conclusions raised by extended theories in such strong gravity regimes. In fact, the resolution of the generalised system of Tolman-Oppenheimer-Volkoff equations for paradigmatic scalar-tensor theories has indeed proved first of all, the existence of solutions for a wide range of the model parameters, and second, the possibility to overcome General Relativity upper bounds for the allowed mass. More to the point, in [72] we proved how for the viable parameters space of $\alpha R^{2}$ models the $f(R)$ predictions for the neutron stars maximal mass ranged from $2-2.6 M_{\odot}$ and therefore was in agreement with observed stars. Analogous results were found for the popular Hu-Sawicki $f(R)$ model in [68].

In addition, we have confirmed how a careful matching of the interior and exterior spacetime regions implies that $(i)$ in the context of $f(R)$ theories, we have shown the existence of exterior static and spherically symmetric physical solutions differing from the usual Schwarzschild solution; (ii) the asymptotic gravitational mass to be unbounded for sufficiently large values of $\alpha$; and (iii) the Ricci scalar is not identically zero at the surface of the star although (for $\alpha>0$ ) it is rapidly suppressed by damped oscillations and asymptotic flatness is eventually recovered. Qualitatively, our results showed how the extra scalar mode present in $f(R)$ theories can be thought of as an additional energetic content around the star. More precisely, the existence of this mode seems to prevent the gravitational collapse and increases the asymptotically observed gravitational mass as shown in the Figure 3. Consequently, neutron star mergers in the context of scalar-tensor theories, would host a higher available energy than that in their Einsteinian counterparts. This may lead to a more efficient and detectable emission of gravitational waves [68,76-81]. Obviously, in the recently opened era of gravitational-wave physics, there is still some room $[82,83]$ for such phenomena to help unveil the real nature of the gravitational interaction. Nevertheless, at the present time our lack of understanding of the strong interaction sector remains larger than the corrections arising from the modifications of gravity. Therefore, current observational data cannot provide much information on the underlying theory of gravity unless complementary tests both in the strong grativity regime and particle physics are performed.

Acknowledgments: AdlCD acknowledges financial support from projects FPA2014-53375-C2-1-P Spanish Ministry of Economy and Science, FIS2016-78859-P European Regional Development Fund and Spanish Research Agency (AEI), CA16104 and CA15117 COST Actions EU Framework Programme Horizon 2020, University of Cape Town Launching Grants Programme and National Research Foundation grants 99077 2016-2018, Ref. No. CSUR150628121624, 110966 Ref. No. BS170509230233 and the NRF Incentive Funding for Rated 
Researchers (IPRR), Ref. No. IFR170131220846. The author would like to thank the organisers of the IV Cosmology and the Quantum Vacuum meeting (Segovia, Spain, Sept. 2017) for the opportunity to present these results.

Conflicts of Interest: The author declares no conflict of interest.

\section{References}

1. Riess, A.G.; Filippenko, A.V.; Challis, P.; Clocchiatti, A.; Diercks, A.; Garnavich, P.M.; Gilliland, R.L.; Hogan, C.J.; Jha, S.; Kirshner, R.P.; et al. Observational evidence from supernovae for an accelerating universe and a cosmological constant. Astron. J. 1998, 116, 1009.

2. Riess, A.G.; Strolger, L.G.; Tonry. J.; Casertano, S.; Ferguson, H.C.; Mobasher, B.; Challis, P.; Filippenko, A.V.; Jha, S.; Li, W.; et al. Type Ia Supernova Discoveries at $\mathrm{z}>1$ from the Hubble Space Telescope: Evidence for Past Deceleration and Constraints on Dark Energy Evolution. Astrophys. J. 2004, 607, 665.

3. Perlmutter, S.; Aldering, G.; Goldhaber, G.; Knop, R.A.; Nugent, P.; Castro, P.G.; Deustua, S.; Fabbro, S.; Gooba. A.; Groom, D.E.; et al. Measurements of $\Omega$ and $\Lambda$ from 42 High-Redshift Supernovae. Astrophys. J. 1999, 517, 565.

4. Abbott, B.P.; Abbott, R.; Abbott, T.D.; Abernathy, M.R.; Acernese, F.; Ackley, K.; Adams, C.; Adams, T.; Addesso, P.; Adhikari, R.X.; et al. Tests of general relativity with GW150914. Phys. Rev. Lett. 2016, 116, 221101.

5. Racusin, J.L.; Burns, E.; Goldstein, A.; Connaughton, V.; Wilson-Hodge, C.A.; Jenke, P.; Blackburn, L.; Briggs, M.S.; Broida, J.; Camp, J. Searching the Gamma-ray sky for Counterparts to Gravitational Wave Sources: Fermi Gamma-ray Burst Monitor and Large Area Telescope Observations of LVT151012 and GW151226. Astrophys. J. 2017, 835, 82.

6. Hughey, B.; Zanolin, M.; Szczepanczyk, M.; Gill, K. GW151226: Observation of Gravitational Waves from a 22-Solar-Mass Binary Black Hole Coalescence. Phys. Rev. Lett. 2016, 116, 241103.

7. Abbott, B.P.; Abbott, R.; Abbott, T.D.; Acernese, F.; Ackley, K.; Adams, C.; Adams, T.; Addesso, P.; Adhikari, R.X.; Adya, V.B.; et al. GW170104: Observation of a 50-Solar-Mass Binary Black Hole Coalescence at Redshift 0.2. Phys. Rev. Lett. 2017, 118, 221101.

8. Abbott, B.P.; Abbott, R.; Abbott, T.D.; Acernese, F.; Ackley, K.; Adams, C.; Adams, T.; Addesso, P.; Adhikari, R.X.; Adya, V.B.; et al. GW170608: Observation of a 19-solar-mass Binary Black Hole Coalescence. Astrophys. J. 2017, 851, L35.

9. Abbott, B.P.; Abbott, R.; Abbott, T.D.; Acernese, F.; Ackley, K.; Adams, C.; Adams, T.; Addesso, P.; Adhikari, R.X.; Adya, V.B.; et al. GW170814: A Three-Detector Observation of Gravitational Waves from a Binary Black Hole Coalescence. Phys. Rev. Lett. 2017, 119, 141101.

10. Abbott, B.P.; Abbott, R.; Abbott, T.D.; Acernese, F.; Ackley, K.; Adams, C.; Adams, T.; Addesso, P.; Adhikari, R.X.; Adya, V.B.; et al. GW170817: Observation of Gravitational Waves from a Binary Neutron Star Inspiral. Phys. Rev. Lett. 2017, 119, 161101.

11. Clarkson, C.; Ellis, G.; Larena, J.; Umeh, O. Does the growth of structure affect our dynamical models of the universe? The averaging, backreaction and fitting problems in cosmology. Rept. Prog. Phys. 2011, 74, 112901.

12. Raccanelli, A.; Bertacca, D.; Pietrobon, D.; Schmidt, F.; Samushia, L.; Bartolo, N.; Doré, O.; Matarrese, S.; Percival, W.J. Testing Gravity Using Large-Scale Redshift-Space Distortions. Mon. Not. Roy. Astron. Soc. 2013, 436, 89-100.

13. Tsagas, C.G.; Challinor, A.; Maartens, R. Relativistic cosmology and large-scale structure. Phys. Rep. 2008, 465, 61-147.

14. De la Cruz-Dombriz, A.; Dobado, A.; Maroto, A.L. On the evolution of density perturbations in $f(R)$ theories of gravity. Phys. Rev. D 2008, 77, 123515.

15. Capozziello, S.; Carloni, S.; Troisi, A. Quintessence without scalar fields. Recent Res. Dev. Astron. Astrophys. 2003, 1, 625 .

16. Nojiri, S.; Odintsov, S.D. Modified gravity with negative and positive powers of curvature: Unification of inflation and cosmic acceleration. Phys. Rev. D 2003, 68, 123512.

17. Carroll, S.M.; Duvvuri, V.; Trodden, M.; Turner, M.S. Is cosmic speed-up due to new gravitational physics? Phys. Rev. D 2004, 70, 043528.

18. De la Cruz-Dombriz, A.; Dobado, A. A $f(R)$ gravity without cosmological constant. Phys. Rev. D 2006, 74, 087501. 
19. De la Cruz-Dombriz, Á.; Dunsby, P.K.S.; Kandhai, S.; Sáez-Gómez, D. Theoretical and observational constraints of viable $f(R)$ theories of gravity. Phys. Rev. D 2016, 93, 084016.

20. De la Cruz-Dombriz, A. Towards Degeneracy Problem Breaking by Large Scale Structures Methods. In Progress in Mathematical Relativity, Gravitation and Cosmology; Springer: Berlin, Germany, 2014; pp. $243-247$.

21. Nojiri, S.; Odintsov, S.D. Unified cosmic history in modified gravity: From F (R) theory to Lorentz non-invariant models. Phys. Rept. 2011, 505, 59.

22. Nojiri, S.; Odintsov, S.D. Introduction to modified gravity and gravitational alternative for dark energy. Int. J. Geom. Meth. Mod. Phys. 2007, 4, 115-146.

23. Capozziello, S.; Faraoni, V. Beyond Einstein Gravity; Springer: New York, NY, USA, 2010.

24. Capozziello, S.; De Laurentis, M. Extended theories of gravity. Phys. Rep. 2011, 509, 167.

25. De la Cruz-Dombriz, A.; Sáez-Gómez, D. Black holes, cosmological solutions, future singularities, and their thermodynamical properties in modified gravity theories. Entropy 2012, 14, 1717.

26. Hehl, F.W.; Von Der Heyde, P.; Kerlick, G.D.; Nester, J.M. General Relativity with Spin and Torsion: Foundations and Prospects. Rev. Mod. Phys. 1976, 48, 393.

27. Hayashi, K.; Shirafuji, T. New General Relativity. Phys. Rev. D 1979, 19, 3524.

28. Wilczek, F. Riemann-Einstein structure from volume and gauge symmetry. Phys. Rev. Lett. 1998, 80, 4851.

29. Obukhov, Y.N. Spectral Geometry of the Riemann-cartan Space-time. Nucl. Phys. B 1983, $212,237$.

30. Benn, I.M.; Dereli, T.; Tucker, R.W. Gauge Field Interactions in Spaces with Arbitrary Torsion. Phys. Lett. 1980, 96, 100.

31. Aldrovandi, R.; Pereira, J.G. Teleparallel Gravity: An Introduction; Springer: Berlin, Germany, 2013; Volume 173.

32. Ferraro, R.; Fiorini, F. Modified teleparallel gravity: Inflation without inflaton. Phys. Rev. D 2007, 75, 084031.

33. Bengochea, G.R.; Ferraro, R. Dark torsion as the cosmic speed-up. Phys. Rev. D 2009, 79, 124019.

34. Linder, E.V. Einstein's Other Gravity and the Acceleration of the Universe. Phys. Rev. D 2010, 81, 127301.

35. Myrzakulov, R. Accelerating universe from F(T) gravity. Eur. Phys. J. C 2011, 71, 1752.

36. De la Cruz-Dombriz, Á.; Farrugia, G.; Said, J.L.; Sáez-Gómez, D. Cosmological reconstructed solutions in extended teleparallel gravity theories with a teleparallel Gauss? Bonnet term. Class. Quant. Grav. 2017, 34,235011

37. De la Cruz-Dombriz, Á.; Dunsby, P.K.S.; Sáez-Gómez, D. Junction conditions in extended Teleparallel gravities. J. Cosmol. Astropart. P. 2014, 1412, 048.

38. Dunsby, P.K.S.; Luongo, O. On the theory and applications of modern cosmography. Int. J. Geom. Meth. Mod. Phys. 2016, 13, 1630002.

39. Busti, V.C.; de la Cruz-Dombriz, Á.; Dunsby, P.K.S.; Sáez-Gómez, D. Is cosmography a useful tool for testing cosmology? Phys. Rev. D 2015, 92, 123512.

40. De la Cruz-Dombriz, Á. Limitations of cosmography in extended theories of gravity. PoS DSU 2016, $2015,007$.

41. Nair, R.; Jhingan, S.; Jain, D. Exploring scalar field dynamics with Gaussian processes. J. Cosmol. Astropart. Phys. 2014, 1401, 5.

42. Capozziello, S.; Farooq, O.; Luongo, O.; Ratra, B. Cosmographic bounds on the cosmological decelerationacceleration transition redshift in $f(R)$ gravity. Phys. Rev. D 2014, 90, 044016.

43. Aviles, A.; Bravetti, A.; Capozziello, S.; Luongo, O. Updated constraints on $f(R)$ gravity from cosmography. Phys. Rev. D 2013, 87, 044012.

44. Capozziello, S.; Luongo, O.; Saridakis, E.N. Transition redshift in $f(T)$ cosmology and observational constraints. Phys. Rev. D 2015, 91, 124037.

45. De la Cruz-Dombriz, A.; Dunsby, P.K.S.; Luongo, O.; Reverberi, L. Model-independent limits and constraints on extended theories of gravity from cosmic reconstruction techniques. J. Cosmol. Astropart. Phys. 2016, $1612,042$.

46. Oppenheimer, J.R.; Volkoff, G.M. On Massive neutron cores. Phys. Rev. 1939, 55, 374.

47. Tolman, R.C. Static solutions of Einstein's field equations for spheres of fluid. Phys. Rev. 1939, 55, 364.

48. Dobado, A.; Llanes-Estrada, F.J.; Oller, J.A. The existence of a two-solar mass neutron star constrains the gravitational constant G_N at strong field. Phys. Rev. C 2012, 85, 012801.

49. Demorest, P.; Pennucci, T.; Ransom, S.; Roberts, M.; Hessels, J. Shapiro Delay Measurement of A Two Solar Mass Neutron Star. Nature 2010, 467, 1081.

50. Barziv, O.; Kaper, L.; van Kerkwijk, M.H.; Telting, J.H.; van Paradijs, J. The mass of the neutron star in vela x-1. Astron. Astrophys. 2001, 377, 925-944. 
51. Rawls, M.L.; Orosz, J.A.; McClintock, J.E.; Torres, M.A.P.; Bailyn, C.D.; Buxton, M.M. Refined Neutron-Star Mass Determinations for Six Eclipsing X-Ray Pulsar Binaries. Astrophys. J. 2011, 730, 25.

52. Munoz-Darias, T.; Casares, J.; Martinez-Pais, I.G. The k-correction for irradiated emission lines in lmxbs: Evidence for a massive neutron star in x1822-371 (v691 cra). Astrophys. J. 2005, 635, 502.

53. Nice, D.J.; Splaver, E.M.; Stairs, I.H.; Loehmer, O.; Jessner, A.; Kramer, M., II; Cordes, J.M. A 2.1 solar mass pulsar measured by relativistic orbital decay. Astrophys. J. 2005, 634, 1242.

54. Antoniadis, J.; Freire, P.C.C.; Wex, N.; Tauris, T.M.; Lynch, R.S.; van Kerkwijk, M.H.; Kramer, M.; Bassa, C.; Dhillon, V.S.; Driebe, T.; et al. A Massive Pulsar in a Compact Relativistic Binary. Science 2013, 340, 6131.

55. Dexheimer, V.A.; Vasconcellos, C.A.Z.; Bodmann, B.E.J. On the Density Dependent Nuclear Matter Compressibility. Phys. Rev. C 2008, 77, 065803.

56. Kobayashi, T.; Maeda, K.I. Relativistic stars in $f(R)$ gravity, and absence thereof. Phys. Rev. D 2008, 78, 064019.

57. Upadhye, A.; Hu, W. Existence of relativistic stars in $f(R)$ gravity. Phys. Rev. D 2009, 80, 064002.

58. Feng, W.X.; Geng, C.Q.; Kao, W.F.; Luo, L.W. Equation of State of Neutron Stars with Junction Conditions in the Starobinsky Model. Int. J. Modern Phys. 2018, 27, 1750186.

59. Teppa Pannia, F.A.; GarcAa, F.; Perez Bergliaffa, S.E.; Orellana, M.; Romero, G.E. Structure of Compact Stars in R-squared Palatini Gravity. Gen. Rel. Grav. 2017, 49, 25.

60. Wojnar, A.; Velten, H. Equilibrium and stability of relativistic stars in extended theories of gravity. Eur. Phys. J. C 2016, 76, 697.

61. Arapoğlu, S.; Çikintoğlu, S.; Yavuz Ekşi, K. Relativistic Stars in Starobinsky gravity with matched asymptotic expansion. Phys. Rev. D 2017, 96, 084040.

62. Katsuragawa, T.; Nojiri, S.; Odintsov, S.D.; Yamazaki, M. Relativistic stars in de Rham-Gabadadze-Tolley massive gravity. Phys. Rev. D 2016, 93, 124013.

63. Fiziev, P.P. A Realistic Model of a Neutron Star in Minimal Dilatonic Gravity. arXiv 2015, arXiv:1506.08585.

64. Hendi, S.H.; Bordbar, G.H.; Eslam Panah, B.; Najafi, M. Dilatonic Equation of Hydrostatic Equilibrium and Neutron Star Structure. Astrophys. Space Sci. 2015, 358, 30.

65. Momeni, D.; Gholizade, H.; Raza, M.; Myrzakulov, R. Tolman-Oppenheimer-Volkoff equations in nonlocal $f(R)$ gravity. Int. J. Mod. Phys. A 2015, 30, 1550093.

66. Zubair, M.; Abbas, G. Some interior models of compact stars in $f(R)$ gravity. Astrophys. Space Sci. 2016, $361,342$.

67. Bakirova, E.; Folomeev, V. Dipole magnetic field of neutron stars in $f(R)$ gravity. Gen. Rel. Grav. 2016, 48, 135.

68. Aparicio Resco, M.; de la Cruz-Dombriz, Á.; Llanes Estrada, F.J.; Zapatero Castrillo, V. On neutron stars in $f(R)$ theories: Small radii, large masses and large energy emitted in a merger. Phys. Dark Univ. 2016, 13, 147-164.

69. Moraes, P.H.R.S.; Arbañil, J.D.V.; Malheiro, M. Stellar equilibrium configurations of compact stars in $f(R, T)$ gravity. J. Cosmol. Astropart. Phys. 2016, 1606, 005.

70. Sharif, M.; Yousaf, Z. Dynamics of spherical stars with structure scalars and $R+\epsilon R^{n} \operatorname{cosmology}$. Can. J. Phys. 2015, 93, 905-911.

71. Sotani, H.; Kokkotas, K.D. Maximum mass limit of neutron stars in scalar-tensor gravity. Phys. Rev. D 2017, 95, 044032.

72. Astashenok, A.V.; Odintsov, S.D.; de la Cruz-Dombriz, A. The realistic models of relativistic stars in $f(R)=R+\alpha R^{2}$ gravity. Class. Quant. Grav. 2017, 34, 205008.

73. Clifton, T.; Dunsby, P.; Goswami, R.; Nzioki, A.M. On the absence of the usual weak-field limit, and the impossibility of embedding some known solutions for isolated masses in cosmologies with $f(R)$ dark energy. Phys. Rev. D 2013, 87, 063517.

74. Nzioki, A.M.; Goswami, R.; Dunsby, P.K.S. Jebsen-Birkhoff theorem and its stability in $f(R)$ gravity. Phys. Rev. D 2014, 89, 064050.

75. Akmal, A.; Pandharipande, V.R.; Ravenhall, D.G. Equation of state of nucleon matter and neutron star structure. Phys. Rev. C 1998, 58, 1804.

76. Damour, T.; Esposito-Farese, G. Tensor-Scalar gravity and binary pulsar experiments. Phys. Rev. D 1996, 54,1474 . 
77. Berti, E.; Barausse, E.; Cardoso, V.; Gualtieri, L.; Pani, P.; Sperhake, U.; Stei, L.C.; Wex, N.; Yagi, K.; Baker, T.; et al. Testing General Relativity with Present and Future Astrophysical Observations. Class. Quant. Grav. 2015, 32, 243001.

78. Koyama, K.; Niz, G.; Tasinato, G. Analytic solutions in non-linear massive gravity. Phys. Rev. Lett. 2011, 107, 131101.

79. Sibandze, D.B.; Goswami, R.; Maharaj, S.D.; Nzioki, A.M.; Dunsby, P.K.S. Scalar Wave Scattering from Schwarzschild Black Holes in Modified Gravity. arXiv 2016, arXiv:1611.06043.

80. Sibandze, D.B.; Goswami, R.; Maharaj, S.D.; Dunsby, P.K.S. Echoes from the Black Holes: Evidence of Higher Order Corrections to General Relativity in Strong Gravity Regime. arXiv 2017, arXiv:1702.04926.

81. Beltran Jimenez, J.; Heisenberg, L.; Olmo, G.J.; Rubiera-Garcia, D. Born-Infeld Inspired Modifications of Gravity. arXiv 2017, arXiv:1704.03351.

82. Ezquiaga, J.M.; Zumalacárregui, M. Dark Energy After GW170817: Dead Ends and the Road Ahead. Phys. Rev. Lett. 2017, 119, 251304.

83. Creminelli, P.; Vernizzi, F. Dark Energy after GW170817 and GRB170817A. Phys. Rev. Lett. 2017, 119, 251302.

(C) 2018 by the authors. Licensee MDPI, Basel, Switzerland. This article is an open access article distributed under the terms and conditions of the Creative Commons Attribution (CC BY) license (http://creativecommons.org/licenses/by/4.0/). 